\title{
Postgraduate Education Corner
}

PULMONARY, CRITICAL CARE, AND SLEEP PEARLS

\section{A 32-Year-Old Woman With Dyspnea, Lung Cysts, and Previous Pneumothoraces}

Todd M. Kolb, MD, PhD; Landon S. King, MD; and David B. Pearse, MD

\section{CHEST 2013; 144(6):1964-1968}

A 32-year-old woman was referred to our clinic for an abnormal chest CT scan. Her primary complaint was progressive exertional dyspnea over 3 months, not accompanied by wheeze, cough, or chest pain during exercise. Similar symptoms 2 years previously had prompted evaluation by a chest physician, and a CT scan identified multiple pulmonary cysts. Because her dyspnea resolved spontaneously, further workup was not pursued.

The medical history was notable for two previous spontaneous pneumothoraces at age 19 years. Neither was associated with the menstrual cycle. She reported a history of childhood asthma and a 10-year history of psoriatic arthritis, initially managed with methotrexate and more recently with etanercept and adalimumab. Yearly purified protein derivative testing was negative.

The patient's mother also had a history of recurrent spontaneous pneumothorax in her 20s. Her maternal grandmother died of metastatic renal cancer. The patient had no smoking history, and her job was relatively sedentary. With the exception of a 4 -month military deployment to the Middle East 10 years previously, her travel history was unremarkable.

\section{Physical Examination Findings}

The vital signs were normal: afebrile; pulse, 80 beats/min; BP, 135/88 mm Hg; oxygen saturation, $99 \%$ on room air. The BMI was $29.6 \mathrm{~kg} / \mathrm{m}^{2}$. Neck and

Manuscript received April 15, 2013; revision accepted June 18, 2013.

Affiliations: From the Division of Pulmonary and Critical Care Medicine, Department of Medicine, Johns Hopkins University School of Medicine, Baltimore, MD.

Correspondence to: Todd M. Kolb, MD, PhD, Division of Pulmonary and Critical Care Medicine, Johns Hopkins University, Johns Hopkins Asthma and Allergy Center, 5501 Hopkins Bayview Circle, Baltimore, MD 21224-6801; e-mail: toddkolb@jhmi.edu

(C) 2013 American College of Chest Physicians. Reproduction of this article is prohibited without written permission from the American College of Chest Physicians. See online for more details. DOI: $10.1378 /$ chest.13-0902 cardiac examinations were unremarkable. Examination of the chest revealed symmetric excursion with inspiration and normal resonance to percussion. Lung auscultation revealed normal expiration and no wheezes, crackles, or rhonchi. Extremity examination showed no peripheral edema or digital clubbing. Skin examination revealed no facial papules, although there were symmetrical areas of erythema and papules on the extensor surfaces of the forearms and shins. The patient noted that these had been present for several years.

\section{Diagnostic Studies}

Chest CT scanning, performed 2 years previously, demonstrated 10 to 20 small, thin-walled cysts located predominantly in the basal and medial lung zones (Fig 1, arrowheads). The upper lungs were relatively spared. There was no other parenchymal abnormality, pathologic lymphadenopathy, or evidence of pulmonary embolism. Repeat chest CT scan, including inspiratory and expiratory images, showed no evidence of air trapping, and the appearance of the cysts was unchanged.

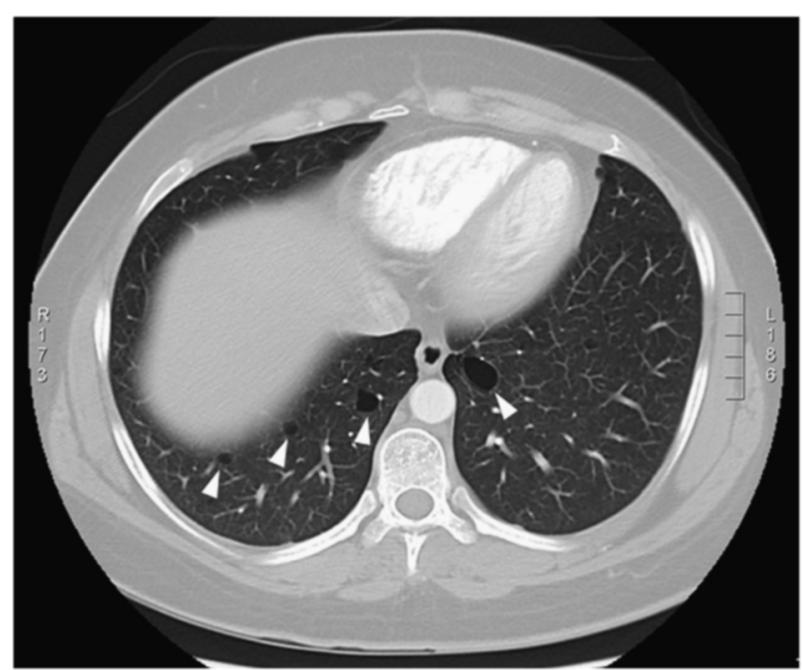

Figure 1. CT scan of the thorax. Arrowheads show cysts in bilateral lower lung zones. 
Pulmonary function testing showed no obstruction $\left(\mathrm{FEV}_{1} / \mathrm{FVC}=80 \%\right)$, normal $\mathrm{FEV}_{1}(3.19 \mathrm{~L}$, $90 \%$ predicted), normal total lung capacity $(5.67 \mathrm{~L}$, $91 \%$ predicted), and normal diffusing capacity ( $25.1 \mathrm{~mL} / \mathrm{mm} \mathrm{Hg} / \mathrm{min}, 88 \%$ predicted). The functional residual capacity (1.22 L, 39\% predicted) and expiratory reserve volume $(0.44 \mathrm{~L}, 32 \%$ predicted $)$ were severely reduced.

Methacholine and exercise provocation challenge were negative. Cardiopulmonary exercise testing was performed, yielding a submaximal study, with the patient reaching only $80 \%$ of her predicted maximal heart rate. Ventilation and perfusion scanning showed no abnormalities. Transthoracic echocardiography showed normal left ventricular structure and function, no significant valvular disease, and a normal pulmonary artery systolic pressure estimate.

What diagnosis explains this patient's abnormal chest CT scan? What study should be conducted next? 
Diagnosis: Birt-Hogg-Dubé syndrome

Study: Folliculin gene sequencing

\section{Discussion}

Birt-Hogg-Dubé syndrome (BHD) is a rare disorder, classically characterized by benign skin hamartomas, multiple lung cysts, spontaneous pneumothorax, and renal cancer. Affected individuals possess a loss of function mutation in the folliculin gene (FLCN) on chromosome 17 . The folliculin protein is highly conserved among species, although its key biologic function has not been clarified fully. Recent identification of folliculin binding partners has implicated the protein in the regulation of cellular metabolism via interaction with the AMP-activated protein kinase.

The actual prevalence of BHD is unknown, although the syndrome has been reported in $>400$ families. FLCN mutations are inherited in an autosomaldominant pattern, although de novo mutations have been described. Although a single FLCN mutation appears to be adequate for skin or pulmonary disease, loss of heterozygosity has been demonstrated in BHD-associated renal cancers, identifying FLCN as a classic tumor suppressor gene. Additional genetic or environmental BHD risk factors have not been described. There are no reported links between BHD and psoriatic arthritis or the treatment regimen described for this patient.

Heterogeneity in the clinical presentation of BHD may contribute to challenges in making this diagnosis. Classic skin findings of BHD include multiple benign follicle hamartomas (fibrofolliculomas and trichodiscomas) on the face, neck, and upper torso. These flesh-colored, dome-shaped papules generally develop in the early 20 s, and are highly prevalent in patients with BHD $(>90 \%)$. However, they are not required to make the diagnosis, because up to $25 \%$ of FLCN mutation carriers have no obvious skin findings. Lung findings, including parenchymal cysts and spontaneous pneumothorax, may provide the earliest clue to the diagnosis. Present in $80 \%$ to $90 \%$ of patients with BHD, parenchymal cysts are localized in the medial and lower lung zones adjacent to interlobular septa or visceral pleura. The number, appearance, and distribution of these cysts can help distinguish BHD from other cystic lung diseases. For example, in contrast to the lung cysts observed in lymphangiomyomatosis, BHD cysts are frequently fewer, less circular, larger, and less widely distributed. In general, BHD cysts are not associated with functional lung abnormalities. These cysts may come to the attention of chest physicians during evaluation for spontaneous pneumothorax, which occurs in approximately $25 \%$ of FLCN mutation carriers. Spontaneous pneumo- thorax in patients with BHD most commonly occurs in the third or fourth decade, and recurrent pneumothorax is common. Renal malignancies are the most concerning manifestation of BHD and are frequently multiple or bilateral at the time of diagnosis. The prevalence of renal cancers in patients with BHD has been estimated at $27 \%$ to $34 \%$, and the mean age at diagnosis is approximately 50 years. In general, these lesions arise much later than the skin or pulmonary manifestations, possibly because of the need for a "second hit" in FLCN.

Diagnostic criteria have been proposed by the European BHD Consortium (Table 1). In the absence of skin findings, identification of a pathogenic FLCN mutation is diagnostic. Clinically available sequence analysis includes the entire coding region and has a reported sensitivity of $88 \%$. Genetic testing should include proband counseling and screening of potentially affected family members. Nearly 150 unique FLCN mutations have been identified. Novel mutations should be reported to one of two publicly accessible BHD mutation databases to promote further understanding of potential genotype-phenotype relationships. Providers may also consider referring patients to an ongoing trial designed to specifically assess these relationships.

Limited information exists to describe the clinical course of BHD. Fibrofolliculomas are reported to increase in size and number over time. Lung findings have not been associated with progressive pulmonary decline or increased mortality. Renal cancer in patients with BHD frequently includes multiple or bilateral tumors, potentially causing significant morbidity. However, metastatic disease has been rarely described, and associated mortality needs further clarification through prospective analysis.

Limited treatment options exist for skin manifestations, and patients with BHD who wish to discuss management options should be referred to an experienced dermatologist. Reported improvements with

\section{Table 1-European BHD Consortium Proposed Diagnostic Criteria}

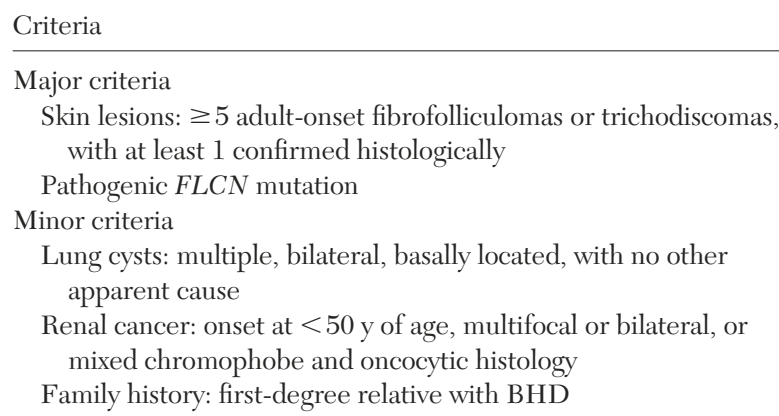

Skin lesions: $\geq 5$ adult-onset fibrofolliculomas or trichodiscomas, with at least 1 confirmed histologically

Pathogenic FLCN mutation

Minor criteria

Lung cysts: multiple, bilateral, basally located, with no other apparent cause

Renal cancer: onset at $<50 \mathrm{y}$ of age, multifocal or bilateral, or mixed chromophobe and oncocytic histology

Family history: first-degree relative with BHD

One major or two minor criteria are needed for a BHD diagnosis. $\mathrm{BHD}=$ Birt-Hogg-Dubé syndrome. 
laser ablation and shave/cautery have been temporary, and topical therapies have proven ineffective. Management of spontaneous pneumothorax in patients with BHD should be consistent with the recommended guidelines for spontaneous pneumothorax in unaffected individuals. Symptoms (breathlessness) should be the primary indication for pleural space evacuation. All patients with BHD should be advised to seek immediate medical attention for acute dyspnea or chest pain, and the risks associated with pressurized environments should be discussed. Patients with other chronic respiratory symptoms (as in the case described here) should undergo further symptom-appropriate evaluation, given the generally preserved lung function in BHD. Smoking has not been associated with pneumothorax development in BHD to date, although we would strongly advocate for smoking cessation in patients with BHD, given (1) the reported association with primary spontaneous pneumothorax; (2) the increased risk of developing smoking-related lung diseases, which could, in turn, increase the severity/consequence of spontaneous pneumothorax; and (3) the increased risk of renal cancer in smokers. Finally, renal cancer screening is a critical component of BHD management. However, at present, there is no consensus recommendation for modality or interval. These choices must balance the considerations of sensitivity, cost, and radiation exposure, given the potential need for extended surveillance in younger individuals. One proposed algorithm recommends initial MRI screening at age 20, with renal ultrasound yearly thereafter. Management of BHD-associated renal cancers should be in accordance with standard staging algorithms. As with other familial renal cancer syndromes, nephronsparing surgery is recommended when lesions exceed $3 \mathrm{~cm}$ in size.

\section{Clinical Course}

The patient's history of recurrent spontaneous pneumothorax and chest radiographic findings raised suspicion for BHD. Although she lacked the classic skin findings, her family history, which included recurrent spontaneous pneumothorax in her mother and renal malignancy in her maternal grandmother, further supported the diagnosis. The patient was offered FLCN sequence analysis after discussing the risks and benefits of genetic testing. Sequence analysis identified a previously undescribed mutation in exon 6 (d.453delG), predicted to encode a frameshift and subsequent premature stop codon. She was referred to our genetic medicine clinic for additional counseling and for coordination of screening for her family members. Sequence analysis revealed the identical mutation in the patient's mother. The novel mutation was reported to the Leiden Open Variation Database (http://grenada.

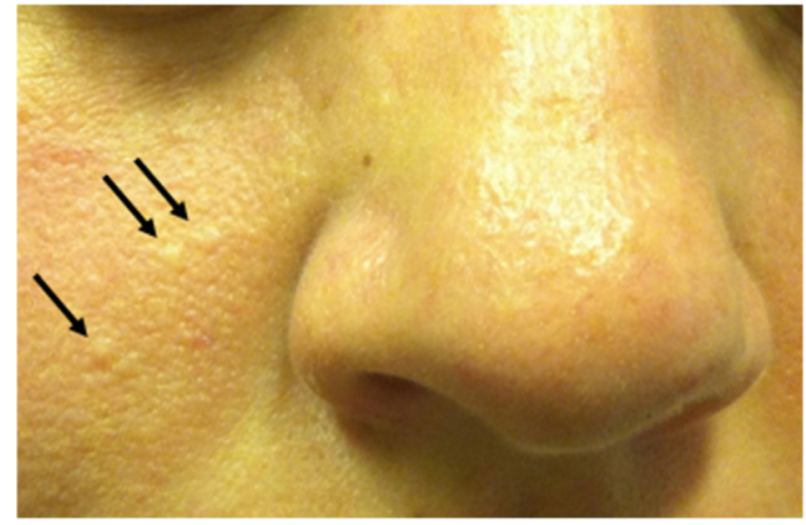

FIGURE 2. Multiple flesh-colored papules (arrows), consistent with early fibrofolliculomas.

lumc.nl/LOVD2/shared1). The patient underwent screening abdominal MRI that showed no renal abnormalities. She was referred to a dermatologist with extensive experience in BHD for evaluation of the chronic skin changes on her forearms and shins, which were not consistent with BHD. However, on follow-up in our clinic approximately 6 months later, new facial papules, more consistent with characteristic fibrofolliculomas, were observed (Fig 2, arrows). The cause of her dyspnea remains unclear, although the results of her cardiopulmonary exercise testing and the reduced expiratory reserve volume and functional residual capacity suggested that deconditioning and obesity were contributors. A trial of weight loss and increased physical activity was recommended.

\section{Clinical Pearls}

1. The differential diagnosis for spontaneous pneumothorax in patients with lung cysts includes BHD, even when skin findings are absent.

2. Lung cysts in BHD are generally sparse, localized to medial and lower lung zones, and not associated with altered lung function.

3. FLCN gene sequencing can be used to definitively diagnose BHD when fibrofolliculomas are absent.

\section{ACKNOWLEDGMENTS}

Financial/nonfinancial disclosures: The authors have reported to CHEST that no potential conflicts of interest exist with any companies/organizations whose products or services may be discussed in this article.

Other contributions: CHEST worked with the authors to ensure that the Journal policies on patient consent to report information were met.

\section{Suggested READINGS}

National Institutes of Health Clinical Center. Genetic analysis of Birt-Hogg-Dube syndrome and characterization of predisposition to kidney cancer. NCT00033137. ClinicalTrials.gov. 
Bethesda, MD: National Institutes of Health; 2002. http:// clinicaltrials.gov/ct2/show/NCT00033137. Updated July 9, 2013.

Zbar B, Alvord WG, Glenn G, et al. Risk of renal and colonic neoplasms and spontaneous pneumothorax in the Birt-HoggDubé syndrome. Cancer Epidemiol Biomarkers Prev. 2002; 11(4):393-400.

Toro JR, Pautler SE, Stewart L, et al. Lung cysts, spontaneous pneumothorax, and genetic associations in 89 families with Birt-Hogg-Dubé syndrome. Am J Respir Crit Care Med. 2007; 175(10):1044-1053.

Toro JR, Wei M-H, Glenn GM, et al. BHD mutations, clinical and molecular genetic investigations of Birt-Hogg-Dubé syndrome: A new series of 50 families and a review of published reports. J Med Genet. 2008;45(6):321-331.
Menko FH, van Steensel MAM, Giraud S, et al; European BHD Consortium. Birt-Hogg-Dubé syndrome: diagnosis and management. Lancet Oncol. 2009;10(12):1199-1206.

Kunogi M, Kurihara M, Ikegami TS, et al. Clinical and genetic spectrum of Birt-Hogg-Dube syndrome patients in whom pneumothorax and/or multiple lung cysts are the presenting feature. J Med Genet. 2010;47(4):281-287.

Tobino K, Hirai T, Johkoh T, et al. Differentiation between Birt-Hogg-Dubé syndrome and lymphangioleiomyomatosis: Quantitative analysis of pulmonary cysts on computed tomography of the chest in 66 females. Eur J Radiol. 2012;81(6): 1340-1346.

Furuya M, Nakatani Y. Birt-Hogg-Dube syndrome: Clinicopathological features of the lung. J Clin Pathol. 2013;66(3):178-186. 